\title{
Smooth Al nanolayers deposition on sapphire and quartz substrates
}

\author{
Piotr Wróbel, ${ }^{1}$ Tomasz Stefaniuk, ${ }^{1}$ Arkadiusz Ciesielski, ${ }^{* 2}$ and Tomasz Szoplik ${ }^{1}$ \\ ${ }^{1}$ Faculty of Physics, University of Warsaw, Pasteura 7, 02-093 Warszawa, Poland \\ ${ }^{2}$ Nanostructures Engineering, Faculty of Physics, University of Warsaw, Hoża 69, 00-681 Warszawa, Poland
}

Received May 14, 2013; accepted June 24, 2013; published June 30, 2013

\begin{abstract}
We report on the roughness of $70 \mathrm{~nm}$ thick $\mathrm{Al}$ films deposited on fused silica and sapphire substrates using an e-beam physical vapour deposition (PVD) system with exact control of substrates temperature. Before evaporation both types of epi-polished substrates are cleaned using low-energy Argon ion bombardment. The roughness of films deposited at 450, 297, 170 and $92 \mathrm{~K}$ is measured with an atomic force microscope (AFM). The lowest average root mean square (RMS) roughness of $0.98 \mathrm{~nm}$ is obtained for a $70 \mathrm{~nm} \mathrm{Al} \mathrm{film} \mathrm{on}$ sapphire deposited at $170 \mathrm{~K}$
\end{abstract}

Rapid development of plasmonics creates a growing interest in techniques leading to the reduction of plasmon scattering losses on metal-dielectric interfaces [1]-[3]. The decrease of surface roughness and interface irregularities should allow for building optical transmission lines with long range surface plasmons as signal carriers [4].

Most of the research in the field is devoted to the properties of silver layers; however, more lossy aluminium/dielectric interfaces are interesting because of wide applications. Studies on Al films are motivated by the use of aluminium in optoelectronics, solar cells and in tapered-fiber metal-coated probes for scanning near field optical microscopes (SNOM) [5]-[8].

In a compilation of Drude model parameters [9] for the optical constants of metals in the infrared and far infrared, the authors claimed that in the case of $\mathrm{Al}$, the free electron model and consequently plasma frequency, damping frequency, dc resistivity, and optical frequency resistivity values should be valid even in the visible range under the condition that the $\mathrm{Al}$ layer is smooth. The relation between surface morphology and scattering of plasmons on thin Al films continues to be of interest [10][13]. Liu et al. [10] reported the deposition of $\mathrm{Al}$ on an $\mathrm{Si}(111)$ wafer at $145 \mathrm{~K}$ and a base pressure of $10^{-10}$ Torr. Starting from 4 monolayers, Al films became atomically flat with RMS $=0.05 \mathrm{~nm}$. Cheng and Lee [12] simulated 3D molecular dynamics of $\mathrm{Al}$ nanolayer growth to determine the dependence of surface roughness on temperature of substrates. Simulations performed for the temperature range $300-700 \mathrm{~K}$ showed that the smallest roughness of an $\mathrm{Al}$ film is achieved at temperatures 450 $600 \mathrm{~K}$. In [10] and [11] plasmons in a few monolayers thin Al films on silicon were studied to measure dispersion as

*E-mail: ac294166@okwf.fuw.edu.pl well as interaction of surface and interface excitations, respectively.

In this paper we report on the roughness of $70 \mathrm{~nm}$ thick Al layers deposited on liquid nitrogen (LN2) cooled fused silica and sapphire substrates using an e-beam Lesker PVD75 system with exact control of temperature of substrates. Non-transparent in the visible range Al films of our interest are used as a metal coating in SNOM probes. The roughness of films deposited at 450, 297, 170 and $92 \mathrm{~K}$ is measured with an atomic force microscope (AFM).

We use double-sided epi-polished substrates of sapphire with c-plane (0001) orientation and nominal RMS $=0.2 \mathrm{~nm}$ roughness as well as made those of fused silica, having nominal roughness RMS of $\leq 0.3 \mathrm{~nm}$.

The roughness of deposited metal films depends on substrate surface contamination, therefore we use wet chemical cleaning and dry etching with Ar ions from Kaufman ion source. To avoid surface erosion due to the knocking of atoms from the crystal lattice nodes, we use low-energy $150 \mathrm{~V}$ ions exposure lasting 30 seconds. This method of pre-cleaning substrates was chosen on the basis of earlier experiments with the deposition of silver films [14].

There are several physical processes influencing the roughness of metal films evaporated in PVD systems. An atom arriving onto a low temperature substrate loses quickly its high kinetic energy and moves only a limited diffusion distance. Thus island growth should be reduced with decreasing substrate temperature. Too large a difference between the room temperature at which the sample is characterized and that of the initially cooled substrate creates a problem of intrinsic stresses in samples due to a mismatch of thermal expansion coefficients of materials involved. The stresses may result in $\mathrm{Al}$ film cracking.

We choose room temperature for deposition to have the reference roughness, where the thermal expansion coefficient mismatch has no influence. To minimize $\mathrm{Al}$ adatom diffusivity, $92 \mathrm{~K}$ is chosen as the lowest temperature available in our LN2 cooled system. In turn, $170 \mathrm{~K}$ is chosen as an intermediate value at which the expansion coefficient mismatch is relaxed but the reduction of $\mathrm{Al}$ adatom diffusivity is still meaningful. In the highest temperature of $450 \mathrm{~K}$ we expect to observe a 
film surface 3D buildup due to island growth and temperature-dependent crystallinity. The temperature of the copper LN2 cooled substrate holder is monitored with two Pt sensors. The use of heat-transfer vacuum cryogenic grease allows for measurement accuracy better than a single Kelvin.
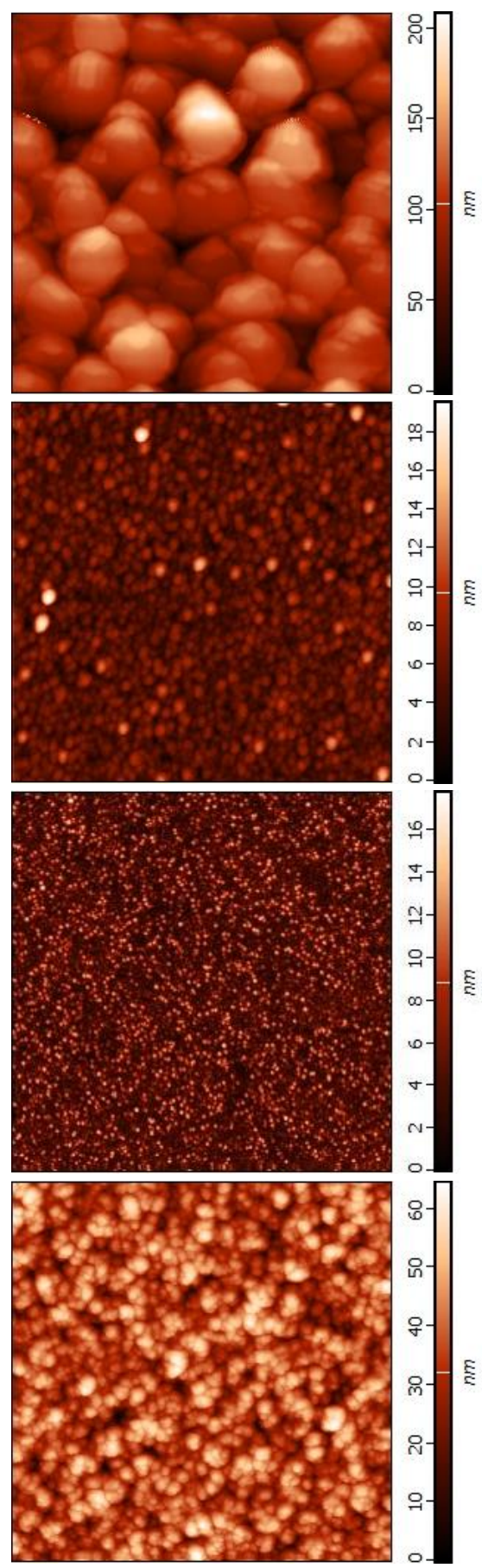

Fig. 1. AFM scans of $3 \times 3 \mu \mathrm{m}^{2}$ area of $70 \mathrm{~nm}$ thick $\mathrm{Al}$ films on quartz substrates deposited at $450 \mathrm{~K}$ (top), $297 \mathrm{~K}$ (upper middle), 170K (lower middle) and 92K (bottom). Note different pseudocolour scales.
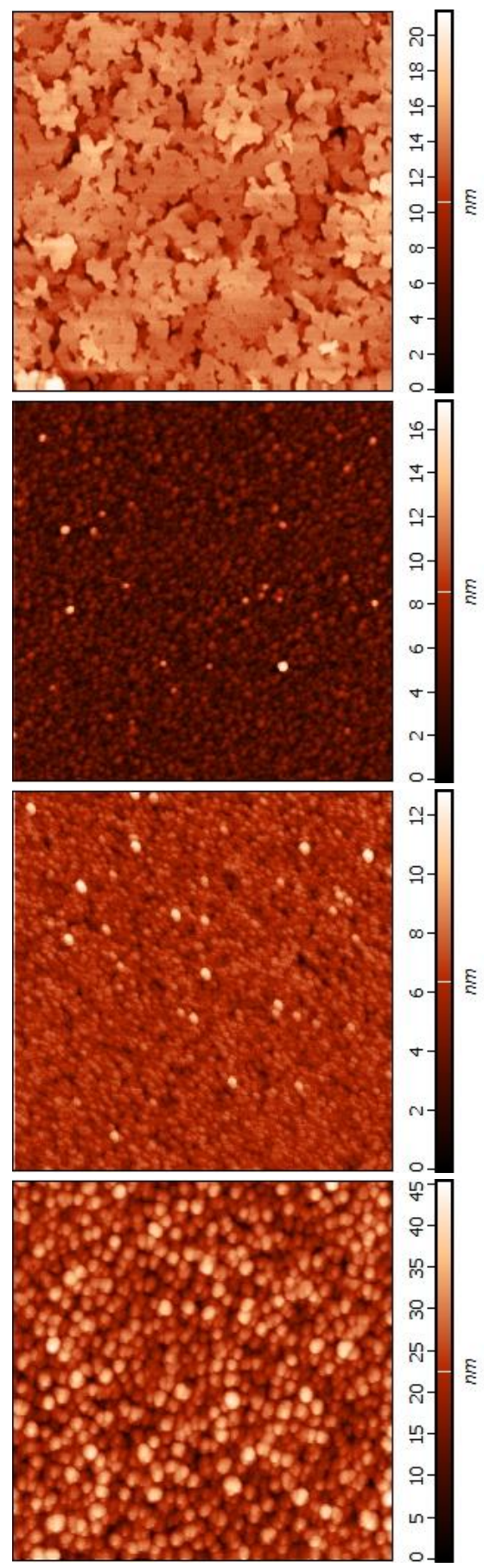

Fig. 2 AFM scans of $3 \times 3 \mu \mathrm{m}^{2}$ area of $70 \mathrm{~nm}$ thick $\mathrm{Al}$ films on sapphire substrates deposited at 450K (top), 297K (upper middle),

170K (lower middle) and 92K (bottom). Note different pseudocolour scales. 
Apart from the substrate temperature, the roughness of Al films depends also on the deposition rate, deposition angle and kinetic energy of depositing atoms. On the basis of earlier experiments we choose the deposition rate equal to $0.1 \mathrm{~nm} / \mathrm{s}$. For substrates of $2 \mathrm{~cm}^{2}$ size and crucible-sample distance equal $0.4 \mathrm{~m}$, the material is deposited perpendicularly to the surface. The kinetic energy of $\mathrm{Al}$ atoms is controlled by the voltage that accelerates electrons, which in the experiment is $6 \mathrm{kV}$.

The thickness of $\mathrm{Al}$ nanolayers is measured with a quartz crystal deposition rate controller calibrated in sequential measurements using NT-MDT AFM and Wyko profilometers.

Figures 1 and 2 show AFM scans of a $3 \times 3 \mu \mathrm{m}^{2}$ area of $70 \mathrm{~nm}$ thick Al films on quartz and sapphire substrates deposited at decreasing temperatures from $450 \mathrm{~K}$ at the top (Figs. 1a and 2a) down to $92 \mathrm{~K}$ at the bottom (Figs.1d and $2 \mathrm{~d}$ ). An amorphous fused-silica substrate is not well wetted by $\mathrm{Al}$ atoms, which results in the growth of huge and separated islands (Fig. 1a). Shown in Fig. 2a, the aluminium film deposited on sapphire at the highest temperature demonstrates the form of small crystal flakes. In a 70nm thick layer it is difficult to assess if a single crystal substrate influences the crystallinity of this deposited metal film. The morphology of samples deposited at two temperatures lower than $450 \mathrm{~K}$ is more uniform. Surface roughness increases when both substrates are cooled down to 92K (Figs. 1d and 2d).

In Table 1, the values of RMS, peak height and average grain size are listed for Al films deposited on fused silica substrates at chosen temperatures. Within the limits of an experimental error, the RMS values measured on films deposited at 170 and $297 \mathrm{~K}$ are similar, which means that at those temperatures of a substrate holder the opposite effects of the expansion coefficient mismatch and reduction of $\mathrm{Al}$ adatom diffusivity neutralize each other.

Table 2 presents the values of RMS, peak height and average grain size in Al films deposited on sapphire substrates at the same four temperatures as above. In Al/sapphire samples both RMS and peak-to-peak values are considerably lower than in $\mathrm{Al} /$ quartz layers. At intermediate temperatures, the RMS values are low due to competing influences of thermal expansion and reduction of $\mathrm{Al}$ adatom diffusivity.

In the samples on substrates of both types, the average grain size evolves in a similar way - we have high granularity at extreme temperatures and low grain size values at intermediate ones.

Our experimental results do not correspond exactly to the simulation of 3D molecular dynamics, which indicates the dependence of surface roughness of $\mathrm{Al}$ films on the temperature of the growth process [12]. The simulations performed for the 300-700 K range have shown that the most smooth Al films are achieved on substrates at 450 $600 \mathrm{~K}$. Our experimental results confirm the existence of a temperature range at which $\mathrm{Al}$ films with low roughness and granularity are evaporated. However, the optimum growth temperatures are seemingly twice lower than those estimated in simulations.

Acknowledgements: This work is supported by the research projects of the Polish National Science Centre UMO-2011/01/M/ST3/05734 and the National Centre for Research and Development \# ARP 178590.

\begin{tabular}{lcccc}
\hline $\mathrm{Al} / \mathrm{SiO}_{2}$ at & $450 \mathrm{~K}$ & $297 \mathrm{~K}$ & $170 \mathrm{~K}$ & $92 \mathrm{~K}$ \\
\hline $\mathrm{RMS}$ [nm] & 26.80 & 1.75 & 2.44 & 9.30 \\
$\begin{array}{l}\text { Peak-to-peak } \\
\text { height [nm] }\end{array}$ & 206.92 & 19.41 & 17.70 & 64.16 \\
$\begin{array}{l}\text { Average grain } \\
\text { size diameter } \\
{[\mathrm{nm}]}\end{array}$ & 259 & 47 & 24 & 102 \\
\hline
\end{tabular}

Table 1. Surface quality parameters of $\mathrm{Al}$ films on quartz substrates measured using AFM.

\begin{tabular}{lcccc}
\hline $\mathrm{Al} / \mathrm{Al}_{2} \mathrm{O}_{3}$ at & $450 \mathrm{~K}$ & $297 \mathrm{~K}$ & $170 \mathrm{~K}$ & $92 \mathrm{~K}$ \\
\hline $\mathrm{RMS}[\mathrm{nm}]$ & 2.07 & 1.28 & 0.98 & 5.94 \\
$\begin{array}{l}\text { Peak-to-peak } \\
\text { height [nm] }\end{array}$ & 21.22 & 17.19 & 12.75 & 45.12 \\
$\begin{array}{l}\text { Average grain } \\
\text { size diameter } \\
{[\mathrm{nm}]}\end{array}$ & 118 & 35 & 59 & 106 \\
\hline
\end{tabular}

Table 2. Surface quality parameters of Al films on sapphire substrates measured using AFM.

\section{References}

[1] D. Sarid, W. Challener, Modern Introduction to Surface Plasmons (Cambridge, Cambridge University Press, 2010).

[2] D.M. Mattox, Handbook of Physical Vapor Deposition (PVD) Processing (Amsterdam, Elsevier, 2010).

[3] J. M. Pitarke, V.M. Silkin, E.V. Chulkov, P.M. Echenique, Rep. Prog. Phys. 70, 1 (2007).

[4] E. Ozbay, Science 311, 189 (2006).

[5] S. Linic, P. Christopher, D.B. Ingram, Nature Materials 10, 911 (2011).

[6] M. Castro-Lopez, D. Brinks, R. Sapienza, N. F. van Hulst, Nano Lett. 11, 4674 (2011).

[7] T.J. Antosiewicz, P. Wróbel, T. Szoplik, Plasmonics 6, 11 (2011)

[8] O.J.F. Martin, M. Paulus, J. Microscopy 205, 147 (2002)

[9] M.A. Ordal, L.L. Long, R.J. Bell, S.E. Bell, R.W. Alexander, Jr., C.A. Ward, Appl. Opt. 22, 1099 (1983). http://www.opticsinfobase.org/ao/abstract.cfm?URI=ao-24-24-4493

[10] H. Liu, Y.F. Zhang, D.Y. Wang, M.H. Pan, J.F. Jia, Q.K. Xue, Surf. Sci. 571, 5 (2004).

[11] Y.H. Yu, Z. Tang, Y. Jiang, K.H. Wu, E.G. Wang, Surf. Sci. 600, 4966 (2006).

[12] Y.Y. Cheng, C.C. Lee, Surface \& Coatings Technol. 203, 918 (2008).

[13] H.J. Qin, Y. Jiang, G.H. Zhang, K.H. Wu, Appl. Phys. Lett. 102, 051605 (2013).

[14] T. Stefaniuk, P. Wróbel, T. Szoplik, Deposition of ultrasmooth silver nanolayers at pressures and temperatures above the sublimation point of water ice, ICTON 2013, NSON Session Proceedings (2013). 